\title{
Coordinated Architecture for Wireless Home Networks
}

\author{
Kandaraj Piamrat and Patrick Fontaine \\ Technicolor \\ 1, avenue de Belle Fontaine \\ 35576 Cesson-Sévigné \\ France \\ \{kandaraj.piamrat, patrick.fontaine\}@technicolor.com
}

\begin{abstract}
Today's services in home networks are no longer limited to basic applications such as email or file transfer but also include multimedia delivery for supporting home entertainment. In addition, wireless network is wide spreading in home as users become mobile and now expect to run their applications in wireless environment the same way they do over wired network. As a consequence, entertainment services should be guaranteed in home wireless networks as well. Ensuring quality of service raises new challenges as open wireless conditions result in instability and vulnerability to all types of interference and disturbance. Especially, IPTV application requires not only throughput but also stability on a wide coverage area and with low packet loss. Therefore, good reception level needs to be guaranteed in the whole house in order to use the highest modulation, and interferences need to be controlled when several transmitters share the same channel. In this paper, we present a new architecture for future home networks, in which multiple access points can be easily deployed on the same channel with coordination established to provide reliable transmission of several IPTV applications in the house. The mechanism is built on top of the DCF and has two main advantages: fully compatible with 802.11 standard and applicable to downlink and uplink streams. For our case study, we use NS-3 to evaluate performances of the Coordinated-APs compared to Single-AP and Distributed-APs approaches, in realistic home environment. The obtained results demonstrate better channel utilization and collision reduction that guarantee four IPTV streams in the coordinated approach. Behaviors at lower layers are presented in order to provide a better understanding of resource utilization. Moreover, discussions about feasibility of the solution in real world scenario are also provided.
\end{abstract}

\section{Categories and Subject Descriptors}

C.2.1 [Network Architecture and Design]: Wireless communication; C.2.2 [Network Protocols]: Applications (SMTP, FTP, etc.); C.2.3 [Network Operations]: Network management

\section{General Terms}

Experimentation, Management, Performance

Permission to make digital or hard copies of all or part of this work for personal or classroom use is granted without fee provided that copies are not made or distributed for profit or commercial advantage and that copies bear this notice and the full citation on the first page. To copy otherwise, or republish, to post on servers or to redistribute to lists, requires prior specific permission and/or a fee.

HomeNets'11, August 15, 2011, Toronto, Ontario, Canada.

Copyright 2011 ACM 978-1-4503-0798-7/11/08...\$10.00.

\section{Keywords}

Home networks, Quality of Service, IPTV application, Wireless Networks, Coordination Function

\section{INTRODUCTION}

In future home networks, the number of connected devices is expected to increase drastically. As shown by recent market studies, the penetration of connected consumer electronic devices such as TVs, smartphones, and tablets are projected to grow $400 \%$ between 2010 and 2015 . Moreover, services in home networks are diverse including multimedia applications such as Internet Protocol Television (IPTV), Video on Demand (VoD), video streaming, Voice over IP (VoIP), online gaming, etc. This raises new challenges in ensuring quality for different applications. In this paper, we focus on the IPTV service provided by network operator. Generally, IPTV is operating on wired network in order to guarantee service quality (with high throughput, stable condition and very low packet loss). It is difficult to imagine running this service on wireless networks even though available in every home today.

Today's home wireless networks operate in the $2.4 \mathrm{GHz}$ ISM band. Only 3 non-overlapping channels are available for the IEEE $802.11 \mathrm{~b} / \mathrm{g}$ standard, which is prone to all types of interference and disturbance. Even if non real-time applications can cope with this, it is not a candidate for IPTV service that requires video packet loss as low as one error in one hour [1]. Here, we will focus on standards IEEE $802.11 \mathrm{a} / \mathrm{n} \mathrm{[2][3]} \mathrm{and} \mathrm{the} \mathrm{incoming} \mathrm{IEEE} \mathrm{802.11ac}$ operating in the $5 \mathrm{GHz}$ band. 19 non-overlapping channels are available on this band which we can assume is enough so that one channel can be assigned per house with proper channel selection algorithm [4]. With several hundreds of Mbps capacity, these networks can drastically improve quality of service (QoS) for IPTV service. Nevertheless, the problem of unstable wireless conditions still remains. The bandwidth requirement of IPTV service requires the usage of the highest modulation, which is more vulnerable to the changing conditions in home environment. Moreover, only one access point (AP) may not be sufficient to cover the whole house with the highest modulation. One solution would be to use several APs scattered throughout the house, following enterprise wireless network solutions like Aruba Networks or Meru Networks. However, home networks gather additional constraints: 
- Must support IPTV application

- Must remain low-cost solution

- Must not require modification in client station

- Must be fully compatible with 802.11 standard

- Must cope with non-cooperative neighbors

In order to keep interference with neighbors to its minimum and to ease management, we assume operating on one channel in the $5 \mathrm{GHz}$ band. However, deploying several APs on the same channel leads to the co-channel interference problem. As the number of stations increases, collision and interference will result in retransmissions and eventually in losses. It can be noticed that even though DCF is not optimal in every network conditions (e.g. hidden node scenario); it is good for burst data traffic under low network load. The objective of this paper is to guarantee a deterministic behavior (i.e. throughput, coverage and error), which is compatible with IPTV service requirement and fairly distributed among every areas in the house. Our goal is not to maximize throughput (because throughput optimization is not enough to guarantee quality for all clients) but to ensure error-free transmission necessary for wireless IPTV service and to reduce collisions of all traffics. Therefore, we propose to deploy multiple access points with coordinated transmissions using a simple Time Division Multiplexing (TDM) mechanism. The implemented scheme is compatible with existing products and provides ease of deployment in current home environment. It requires no change to existing station and can be implemented in AP by driver's update only. The solution is compared to default approaches such as singleAP and distributed-APs approaches, actually deployed in home networks today. Another contribution of this paper also concerns the realistic propagation model that has been simulated using Volcano simulator [5]. It uses ray-tracing simulation method based on real house description. For more detailed of propagation, please refer to [6]. The paper will focus particularly on the behavior of each approach in utilizing the wireless channel.

The remaining of this paper is organized as follows. Section 2 provides some backgrounds on related works. Section 3 presents the coordinated approach. Case studies have been conducted and results of the different approaches are presented in Section 4. Finally, Section 5 concludes the paper and discusses future works.

\section{RELATED WORKS}

According to the literature, there exist two principal approaches to manage the wireless transmission in home or enterprise networks. The first approach is the traditional approach called distributed architecture, in which multiple APs are deployed to cover the target area. These APs are autonomous and have all the functionalities including transmissions of beacons, data, RTS/CTS as well as management and control aspects. We often call this type of AP as Fat AP. The advantage of this approach is the autonomy of the access point and the direct transmission.
However, since APs operate independently to each others, the problem of co-channel interference occurs and the control of global network is difficult.

As another alternative, the centralized architecture has been proposed and consists in using a central controller with multiple access points: APs only implement real-time functionalities such as transmission of the packets, beacons, RTS/CTS, etc. while management and control functions are left to the controller. We often call this type of AP as thin $A P$. The communication between the central controller and the APs is done via a standardized protocol such as CAPWAP [7]. The main advantage of this approach is related to a centralized and therefore more efficient management in terms of load balancing, handover management, etc. However, the problem of co-channel interference still remains.

Several centralized mechanisms have been proposed to cope with the co-channel interference problem. Most of them use coordination in order to manage access to the channel. TCF [8] makes use of DCF-like mechanism to manage transmission of active clients in round-robin manner. However, the scheme can be used only when all stations are within the radio transmission range. In fact, TCF causes size of contention window (CW) to be equal to the number of active stations in order to remove idle slots in the contention period. Therefore, the scheme cannot handle hidden node problem and is not compatible with legacy clients. For enterprise network, CENTAUR [9] has been proposed as hybrid architecture (using centralized approach to handle hidden and exposed nodes and using DCF to handle other traffic). Hence, it is particularly beneficial with hidden and exposed terminal traffic in the downlink path but not much gain in non-hidden and non-exposed terminal downlink. Using fixed backoff and packet staggering for concurrent transmission, it exploits exposed nodes scenario to increase transmission throughput. Using hybrid data path, the scheme is compatible with legacy clients and deal with overhead using decision by epoch. However, the scheme does not coordinate the uplink traffic.

In order to get the advantages and avoid drawbacks of existing works previously described, we deploy a subset of centralized architecture while keeping the implementation and overhead minimized. Our target environment is home networks and solution should be able to implement with ease. For that, the access gateway, which is already the central point of home networks, can host the controller without any additional cost.

\section{PROPOSED ARCHITECTURE}

In this section, we present a new architecture using a coordinated lightweight access point protocol (CLAPP), which inherits from centralized architecture with proposition of coordinated transmission among Basic Service Set (BSS). The coordination can be applied to both downlink (DL) and uplink (UL) transmissions. 


\subsection{Architecture Description}

Our coordinated architecture is composed of wireless LAN controller (WLC), coordinated lightweight access points (CL-AP), and client stations (STA). WLC is the central controller of the wireless network; its main functions are to configure CL-APs and ensure acceptable QoS through air time sharing. To do so, WLC initializes an extended service set (ESS) composed of one BSS per CL-AP on a single channel, manages STA associations among BSSs, and coordinate the transmissions between BSSs. CL-AP is a standard access point (as in 802.11-2007 [10]) except that some management functions are moved into the WLC and additional functions like synchronization or scheduling are implemented in order to operate in a coordinated fashion. STA is a legacy station with support of Quiet Element, which is part of Dynamic Frequency Selection (DFS) procedures described in [10], mandatory in $5 \mathrm{GHz}$ band. Figure 1 depicts different entities in the architecture. The CL-APs, located in different rooms of the house/building, are connected to the wired network (Ethernet and/or PowerLine and/or MoCA for example). Several STAs are also dispatched among several rooms. Each of them is associated to one CL-AP. STA can be embedded in Laptop PC, Wi-Fi tablet, Mobile phone, etc. Communications among them are described in section 3.2.

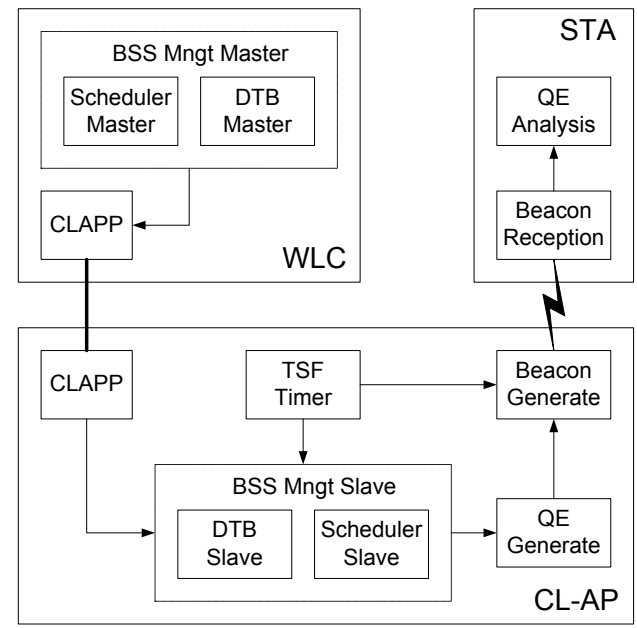

Figure 1. Coordinated architecture.

\subsection{Coordination mechanism description}

First of all, it is worth mentioning that the coordination is built on top of the DCF. The coordination is a sub-optimal solution in terms of throughput but with the objective of reducing the collision of packets that are responsible for packet errors, especially in case of hidden nodes. As the whole set of APs and STAs operates in the same channel, it constitutes a collision domain of relatively large number of transmitters. The coordination mechanism is introduced to act as a protection between BSSs, therefore reducing the collision domain. As the wireless channel remains a common resource, the sharing is achieved with a simple TDM. The following describes the implementation of the mechanism valid for downlink (DL) and uplink (UL) transmissions and compatible with 802.11a client STAs. It is composed of two main functions: synchronization between BSSs and temporal slot allocation.

\subsubsection{Synchronization}

First, CL-APs need to get a common time reference from WLC. The synchronization is achieved through the distribution system independently of the application layer via IEEE1588/Precision Time Protocol [11] (producing a system common clock) plus a Distributed Time Base (DTB) layer (providing a common reference counter between DTB master in WLC and all DTB slaves in CL-APs). Real measurement was conducted with software implementation

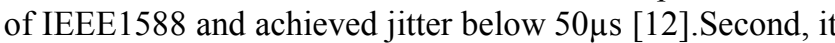
is necessary to translate the timing information from the WLC into a timing reference of each BSS; this is achieved by translating the DTB into TSF timer maintained by each CL-AP. All STAs associated to a CL-AP are synchronized via the Timestamp field in Beacon frames.

\subsubsection{Slot allocation}

The coordinated function is implemented using time division multiple access (TDMA) mechanism. The duration of the temporal slot (sub-frame), which is allocated to each BSS is proportional to the bandwidth needs of its associated stations. Therefore, we obtain dynamic and fair allocations among APs in terms of traffic load. An example of superframe definition and sub-frame allocation for each CL-AP of the ESS is illustrated in Figure 2a. Each BSS uses the standard CSMA/CA to gain access to the medium in its subframe. The protection between BSSs is based on Quiet Element (QE) usage. The QEs enable a CL-AP to prevent any transmission of its associated STA during sub-frames allocated to other BSS, without preventing other BSS to transmit. It can be noticed that our scheme is fully compatible with QoS management of different traffic categories within one BSS, for example, the standard Enhanced Distributed Channel Access (EDCA) mechanism.

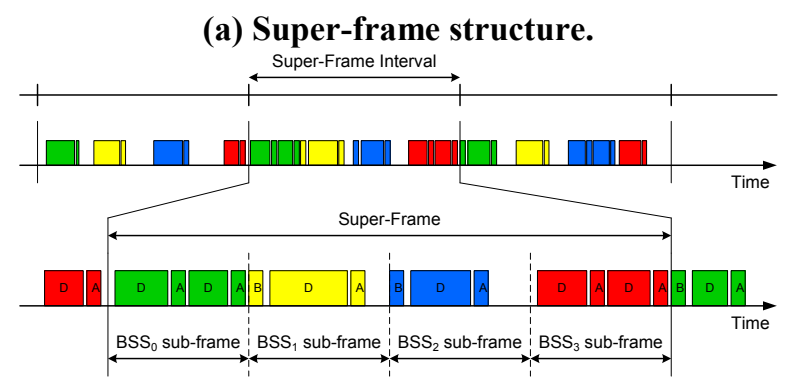

(b) Quieting BSS outside its sub-frame.

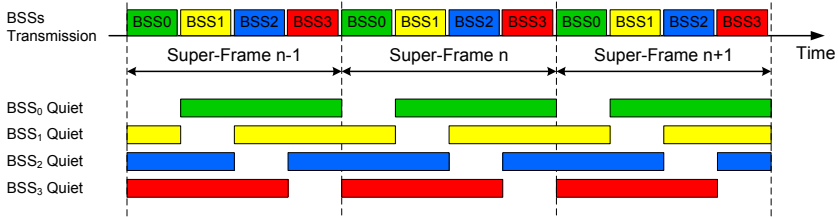

Figure 2. Coordination mechanism. 
The WLC sends the frame definition to each active BSS via the CLAPP. Each scheduler slave in the CL-AP decodes the frame definition and set the NAV outside its sub-frame, therefore preventing itself from any collision with other BSSs. It also translates the frame definition in QEs in order to coordinate the STA associated to its BSS. Each STA decodes the beacon that contains the QEs and set the NAV as illustrated in Figure 2b. Other STAs (associated to other BSS) that also decode the beacon will discard the QEs. This behavior is not possible with other control frames of the standard (e.g. RTS or CTS2self). The co-channel interference problem is therefore solved between BSSs.

\section{CASE STUDY}

In this section, we provide a case study of the proposed mechanism comparing to traditional architectures. We use the floor plan of a real house, which represents the typical size of a house in Europe. We describe network topology, simulation setup, and then discuss the results.

\subsection{Network Topology}

In this paper, coverage is defined as the area where packet error rate or PER (before retransmission) is less than $1 \%$ when using 64QAM3/4 modulation. For multi-cell approach, four APs are necessary in order to provide approximately the full house coverage [6]. Figure 3 depicts signal attenuation within the house with four APs. APs' positions are presented using black dots along with their id. In single-cell approach, the AP is located at the position that provides the best coverage (red dot). Stations are fairly positioned inside the rooms at startup, and then move according to a random walk model within a region of $2 \times 2$ $\mathrm{m}^{2}$. Therefore, stations stay close to the same AP and there is no handover in the scenario.
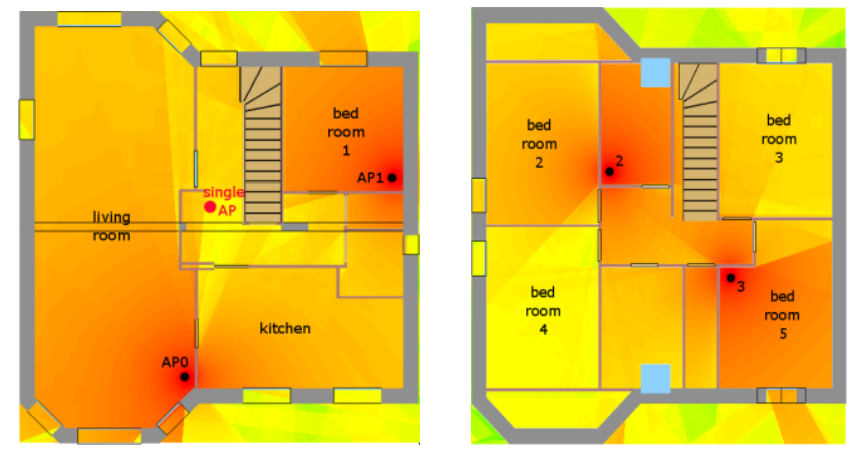

Figure 3. Floor plans with signal attenuation and APs' placement (left: first floor; right: second floor).

\subsection{Simulation Setup}

In order to evaluate the performance of different approaches, we run simulations using NS-3 network simulator [13] with the following configuration. The wireless channel and physical layer models are based on the YANS model [14]. However, since this channel is Gaussian, $19 \mathrm{~dB}$ is added to noise figure in order to model 802.11 Channel B (referring from [15], with $\mathrm{PER}=1 \%$ in
64QAM3/4 1x1). In addition, we deploy realistic loss propagation model, using the ray-tracing propagation simulation with wall attenuation to calculate the signal attenuation between the source and destination nodes [6]. For all scenario, we use IEEE 802.11a [2] operating at $5 \mathrm{GHz}$ channel 36 with bandwidth $20 \mathrm{MHz}$ and AARF-CD [16] for rate control. Table 1 summarizes the configuration.

Table 1. NS-3 Configuration

\begin{tabular}{|c|c|}
\hline Standard & IEEE $802.11 \mathrm{a}$ \\
\hline Frequency Channel & $5 \mathrm{GHz}, 20 \mathrm{MHz}$ bandwidth \\
\hline Noise Figure & $7+19 \mathrm{~dB}$ \\
\hline Energy Detection threshold & $-82 \mathrm{dBm}[10]$ \\
\hline CCA busy threshold & $-62 \mathrm{dBm}[10]$ \\
\hline Output power & $15 \mathrm{dBm}$ \\
\hline Tx and Rx antenna gains & $2 \mathrm{dBi}$ \\
\hline Rate control algorithm & AARF-CD \\
\hline
\end{tabular}

According to our simulations, the network saturation is around 24 Mbps when using 64QAM3/4. Therefore, we selected a basic scenario with four IPTV flows of $4 \mathrm{Mbps}$. This corresponds to realistic scenario where users are watching four channels in different rooms (living room, bedroom 1, bedroom 3, bedroom 5). Since IPTV traffic is CBR [17], we generate CBR traffic of 4 Mbps with 1316bytes packets. In that case, the IPTV service requirement (one error in one hour) corresponds to a video packet loss rate less than $10^{-7}$. In order to demonstrate impact of the uplink transmission, we also extend the scenario to generate four uplink transmissions (1 Mbps each), which could represent the wireless video surveillance applications emerging in home networks today. All flows start at 2.5 seconds after simulations begin and stop at 4 seconds before the end of simulation. For coordinated and distributed approaches, each downlink or uplink flow is associated to one AP to avoid problem of unbalanced traffic, which can bias the analysis of the results.

The super-frame duration is set to $25 \mathrm{~ms}$ in our case study in order to keep throughput and delay compromised. With this duration, the overhead of coordinated approach is less than $0.5 \%$. For post-processing of the results, we use Matlab that can parse pcap files (including radiotap header [18]) and compute necessary statistics.

\subsection{Results}

In this section, we present the results in different aspects; we begin by the overall performance summarized in Table 2 presenting the video packet statistics of the four IPTV streams in (a) single, (b) distributed, and (c) coordinated architectures. The observed metrics are: percentage of packets dropped at AP queue (\%Drop), percentage of retransmitted packets at AP $(\%$ Retx $)$, video packet loss rate $(P L R)$, and finally whether the IPTV service requirement $\left(\mathrm{PLR}<10^{-7}\right)$ can be guaranteed (Result). In each cell of the table, the left value corresponds to the basic scenario with only downlink traffic and the right value corresponds to the extended scenario with both downlink and uplink traffic. 
Table 2. IPTV performance

\begin{tabular}{|c|c|c|c|c|}
\hline Video & \%Drop & \%Retx & PLR & Result \\
\hline IPTV0 & $2.3 / 7.1$ & $0.4 / 2.5$ & $2.3 \mathrm{e}-2 / 7.1 \mathrm{e}-2$ & Failed / Failed \\
\hline IPTV1 & $5.5 / 30.6$ & $0.1 / 3.7$ & $5.5 \mathrm{e}-2 / 3.1 \mathrm{e}-1$ & Failed / Failed \\
\hline IPTV2 & $5.6 / 30.7$ & $7.0 / 7.7$ & $6.0 \mathrm{e}-2 / 3.1 \mathrm{e}-1$ & Failed / Failed \\
\hline IPTV3 & $5.7 / 31.4$ & $4.4 / 6.1$ & $5.8 \mathrm{e}-2 / 3.1 \mathrm{e}-1$ & Failed / Failed \\
\hline
\end{tabular}

(a) Single-AP (DL-only / DL+UL)

\begin{tabular}{|c|c|c|c|c|}
\hline Video & \%Drop & \%Retx & PLR & Result \\
\hline IPTV0 & $0 / 0$ & $0.1 / 6.6$ & $0 / 0$ & Passed / Passed \\
\hline IPTV1 & $0 / 0.004$ & $18.2 / 20.7$ & $2.1 \mathrm{e}-5 / 8.6 \mathrm{e}-5$ & Failed / Failed \\
\hline IPTV2 & $0 / 0$ & $2.5 / 6.4$ & $0 / 0$ & Passed / Passed \\
\hline IPTV3 & $0 / 0$ & $15.6 / 16.1$ & $0 / 1.5 \mathrm{e}-4$ & Passed / Failed \\
\hline
\end{tabular}

(b) Distributed-AP (DL-only / DL+UL)

\begin{tabular}{|c|c|c|c|c|}
\hline Video & \%Drop & \%Retx & PLR & Result \\
\hline IPTV0 & $0 / 0$ & $0 / 7.7$ & $0 / 0$ & Passed / Passed \\
\hline IPTV1 & $0 / 0$ & $0 / 10.9$ & $0 / 0$ & Passed / Passed \\
\hline IPTV2 & $0 / 0$ & $0 / 6.4$ & $0 / 0$ & Passed / Passed \\
\hline IPTV3 & $0 / 0$ & $0 / 7.9$ & $0 / 0$ & Passed / Passed \\
\hline
\end{tabular}

(c) Coordinated-AP (DL-only / DL+UL)

\subsubsection{Single-AP approach}

It can be seen from Table 2 (a) that the single-AP approach cannot guarantee the requirement of any IPTV stream in both basic and extended scenario. The percentage of packet drops in the AP queue is respectively high in the basic scenario and very high with uplink $(5.7 \%$ and $31.4 \%$ resp. on IPTV3). Consequently, the video PLR is also very high $\left(\mathrm{PLR}>10^{-2}\right)$. This is because only one AP cannot guarantee the coverage of the whole house (for this case study, it covers around $50 \%$ of the house [6]).
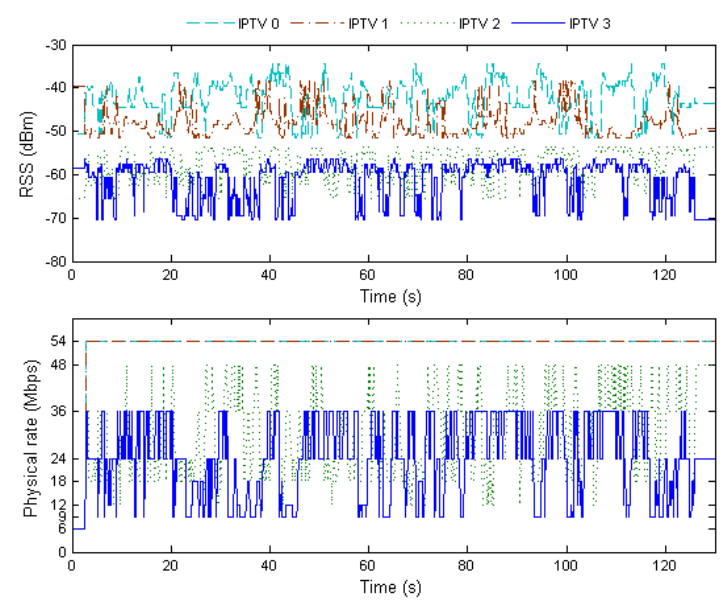

Figure 4. RSS and physical rate for single AP approach

For more details, Figure 4 illustrates the received signal strength (RSS) of the four IPTV clients and the selected modulations by the AP. It can be noticed that flows IPTV0 and IPTV1 get good RSS since the two clients are located on the same floor as the AP and the selected modulation is the highest (54 Mbps rate). Even though, the AP cannot guarantee the required PLR of IPTV. In fact, the two other clients situated on the second floor suffer from lower RSS, and the AP never selects rates higher than $36 \mathrm{Mbps}$ for IPTV3 client. As a result, it takes longer time to transmit packets and affects drastically the performance of all IPTV flows; hence the drops on the AP queue resulting from lack of bandwidth. This illustrates the performance anomaly [19] when stations have different capacities for receiving.

\subsubsection{Distributed approach}

It can be seen from Table 2 (b) that the distributed approach achieves better results than the single-AP approach. In the basic scenario, three out of fours IPTV streams meet the IPTV requirement, and two out of four in the presence of additional uplink streams. The lack of bandwidth issue seen previously has been solved as shown by the \%Drop metric (zero packet drops in the basic and extended scenario). This can be explained by the fact that with multiple APs deployment stations are close to at least one AP and thus the selected modulation can always be the highest (54 Mbps in our case). Therefore, the remaining problem is now on the high percentage of retransmission. In order to illustrate this problem, we focus on the IPTV1 flow $(18.2 \%$ of retransmitted packets), which does not pass the required PLR. Figure 5 shows that the number of retransmissions in distributed approach is high while coordinated approach has zero retransmission. This result confirms the statement in the previous section that using coordination between access points can help avoiding collision. As both approaches run under the same conditions (same AP positions and same stations' mobility), it also points out that all retransmissions in distributed-AP are due to collisions.

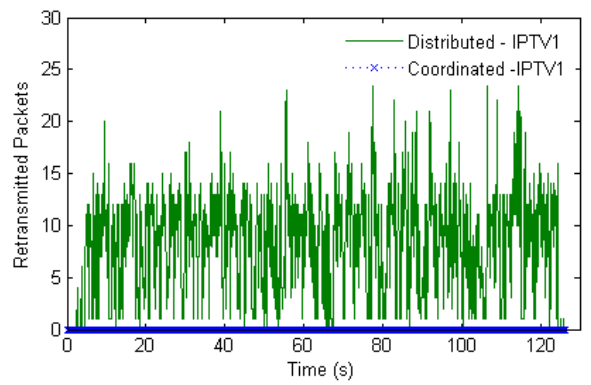

Figure 5. Retransmission comparison (DL only)

\subsubsection{Coordinated approach}

As presented in Table 2 (c), the Coordinated-AP approach fulfills the requirement of the four IPTV flows in both basic and extended scenario. It achieves zero packet loss but also zero retransmitted packet in the basic scenario. This result is naturally obtained with coordinated approach since the coverage issue was solved by the multiple APs deployment and collision of packet cannot occur in coordinated architecture (only one downlink stream is affected to each BSS). The extended scenario is more difficult to handle as uplink stream is added to each BSS. According to the table, zero packet loss can still be achieved; however, the percentage of retransmitted packets increases from the basic 
scenario. It reaches $10.9 \%$ for IPTV1 flow, compared to $20.7 \%$ in the distributed approach. Figure 6 illustrates the retransmission comparison for this IPTV1 in extended scenario. Moreover, it can be noticed that the percentage of retransmitted packets in the distributed approach is doubled compared to the coordinated approach for both IPTV1 and IPTV3 flows. Contrary to distributed approach, the reduction of collision through BSS protection enables coordinated approach to meet the requirement of two additional IPTV flows, in the presence of uplink streams.

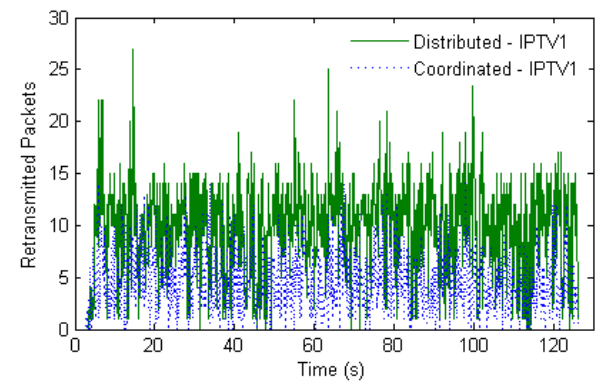

Figure 6. Retransmission comparaison (DL+UL)

\section{CONCLUSIONS AND FUTURE WORKS}

We have proposed a coordinated architecture to handle wireless networks in future home environment. We also demonstrated that one single AP is not sufficient to guarantee the requirement of IPTV service. Using multiple APs in distributed manner can improve performance but can only ensure $75 \%$ and $50 \%$ of IPTV flows in basic and extended scenario respectively. On the contrary, multiple APs using simple coordination mechanism can share the same frequency channel without increasing collision. We observed zero collision in basic scenario and some collisions present in extended scenario. Nevertheless, the collisions can still be recovered with retransmission and quality guarantee on IPTV flows can still be maintained.

As conclusions, our coordinated architecture improves network performance regarding different famous problems. In terms of co-channel interference, the problem is solved among APs since we use the coordinated transmission among them. As for hidden node problem, we coordinated globally the transmission of the APs with central controller. As far as the cost of implementation is concerned, we observe that while the wireless technology progresses, the cost of wireless devices or products such as access points decreases. The coordination mechanism can then be easily implemented in home gateway and AP's driver without modification on legacy client stations. These will facilitate the deployment of multiple APs in future home networks.

For future work, the scheme can be enhanced in two fronts. First, the scheduler can further be refined and time-slot reuse between BSSs (that do not interfere) can be investigated for higher performance. Second, the study can further be extended to area or scenario with intensive usage of wireless applications leading to high number of transmitters in the same channel. We plan to investigate these configurations under IEEE 802.11n.

\section{REFERENCES}

[1] DSL Forum. 2006. Triple-play Services Quality of Experience (QoE) Requirements. Technical Report TR-126.

[2] IEEE 802.11a-1999. Part 11: Wireless LAN Medium Access Control (MAC) and Physical Layer (PHY) Specifications: High-speed Physical Layer in the $5 \mathrm{GHZ}$ band.

[3] IEEE 802.11n-2009. Amendment 5: Enhancements for Higher Throughput.

[4] Smith, G. 2009. Overlapping BSS Analysis of Channel Requirements. IEEE 802.11-08/1470-04-00aa.

[5] SIRADEL. VOLCANO Suite 3.0 2010, url: http://www.siradel.com/1/volcano-software-suite.aspx

[6] Baron, F. 2011. Propagation simulation for NS3. Technicolor Technical Report, CR-RRL-2011-03-0001. url: http://www.thlab.net/ fontainep/ref/

[7] Calhoun, P., Montemurro, M., and Stanley, D. 2009. RFC5415 Control And Provisioning of Wireless Access Points (CAPWAP) Protocol Specification IETF.

[8] Lim, C. and Choi. C.-H. 2004. TDM-based coordination function (TCF) in WLAN for high throughput. In Proceedings of GLOBECOM'04. 5, 3235- 3239.

[9] Shrivastava, V., Ahmed, N., Rayanchu, S., Banerjee, S., Keshav, S., Papagiannaki, K., and Mishra, A. 2009. CENTAUR: realizing the full potential of centralized WLANs through a hybrid data path. In Proceedings of MobiCom'09. 297-308.

[10] IEEE 802.11-2007. Part 11: Wireless LAN Medium Access Control (MAC) and Physical Layer (PHY) Specifications.

[11] IEEE 1588-2002. Standard for a Precision Clock Synchronization Protocol for Networked Measurement and Control Systems.

[12] Correll, K. Barendt, N. and Branicky, M. 2006. Design Considerations for Software Only Implementations of the IEEE 1588 Precision Time Protocol. In Conference on IEEE 1588 Standard for a Precision Clock Synchronization Protocol for Networked Measurement and Control Systems.

[13] The ns-3 network simulator. url: http://www.nsnam.org/

[14] Lacage, M. and Henderson, T. R. 2006. Yet another network simulator. In Proceeding of the 2006 workshop on ns-2: the IP network simulator. 12.

[15] Perahia, E. and Stacey, R. 2008. Next Generation Wireless LANs: Throughput, Robustness, and Reliability in 802.11n. Cambridge University Press.

[16] Maguolo, F., Lacage, M., and Turletti, T. 2008. Efficient collision detection for auto rate fallback algorithm. In Proceeding of ISCC. 25-30.

[17] O'Driscoll, G. 2008. Next Generation IPTV Services and Technologies. Wiley-Interscience.

[18] Radiotap, url: http://www.radiotap.org/

[19] Heusse, M., Rousseau, F., Berger-Sabbatel, G., and Duda, A. 2003. Performance anomaly of $802.11 \mathrm{~b}$. In Proceeding of INFOCOM. 2, 836-843. 